\title{
Implementation of redundancy in the effective regulation of temperature in an incubator
}

\author{
Montasser Damak And Mohamed Ouali \\ CES Laboratory, National School of Engineering of SFAX
}

\begin{abstract}
This paper presents a new mechanism exploiting the redundancy of sensors to ensure reliability in measuring baby incubator parameters where no error is tolerated.

Several research based on the sensors redundancy have been done to several various areas, e.g., aerospace, automotive. However, no investigation is done, based on this technique, to ensure reliable measurement of baby incubator parameters. This paper, precisely, addresses this luck by proposing a new mechanism to precise and efficient measurement of temperature in an incubator. Our way of using redundancy consists in the use of a double of materials sensors with an analytical sensor as well as a time-redundant.

Our new solution has been validated through several tests on baby incubator. Our tests showed that our solution enables the reduction of transitory events and faults in sensors.
\end{abstract}

Keyword-sensor redundancy, time-redundant, supervisory, incubator.

\section{INTRODUCTION}

Recent research has been focused in the use of sensor redundancy to ensure reliable supervisory. While supervisory in medicine is directly involving the lives of human beings, no research is done to ensure reliable baby incubator supervisory. In an incubator, premature baby's life depends on the reliability of the supervisory system. Indeed, the safety of operation in such system depends on the parameters perceived at supervisory. That is, an incubator system acts according to defined orders, which, in their turn, depend on the perceived measurements of the supervisory. Thus, the reliability of the supervisory in such system is tightly related with the safety.

Redundancy is the main method used for fault tolerance. Generally, redundant systems consist in detecting, isolating, and accommodating failures among the redundant elements. The concept of analytical redundancy has been investigated to replace some of the hardware redundancies. To further improve the reliability, the temporal redundancy permits to reduce noise due to transitory events. The major contribution of this paper is to combine these three redundancy methods. First, our solution use two hardware sensors to cope with failures or crashes at physical sensor. In addition, our solution relies on the analytical redundancy to measure the temperature to expect according to the actuators. Using comparison between measurements perceived at hardware sensors and those computed analytically, our solution is able to detect failures at actuators.

Moreover, to cope with noise due the transitory events, our solution relies on the temporal redundancy. We use this mechanism to reduce possible gap between subsequent measurements. In such case, this mechanism defines the new measurement according to the measurements perceived in the past.

Roadmap: This paper is organized as follows. Section II describes our studied environment. More precisely, this section presents the parameters related to baby incubator. Section III gives the details of our solution. Section IV describes the redundancy technics. Section V defines the process monitoring. Section VI expand discussion about conclusions and future work will be made in the last section.

\section{STUDY OF THE INCUBATOR}

The temperature in the incubator is modeled as a first order system with delay identified through several measures that are represented on the following graph: 


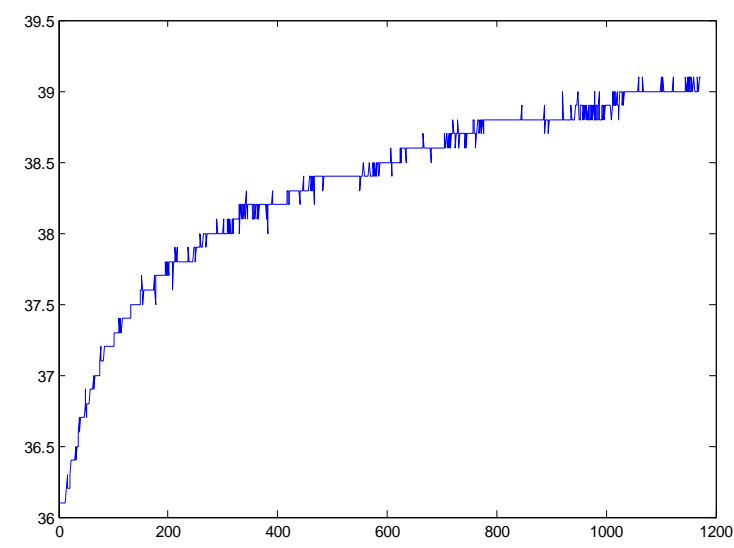

Figure $1:$ Measure of the temperature in the incubator when heating The incubator is a system of first order when heating. The model associated with this latter is : $y(t)=y_{0}+K\left(1-e^{-t / \tau}\right)$ With $y_{0}$ the initial value, $\mathrm{K}=2.560$ and $\tau=330$

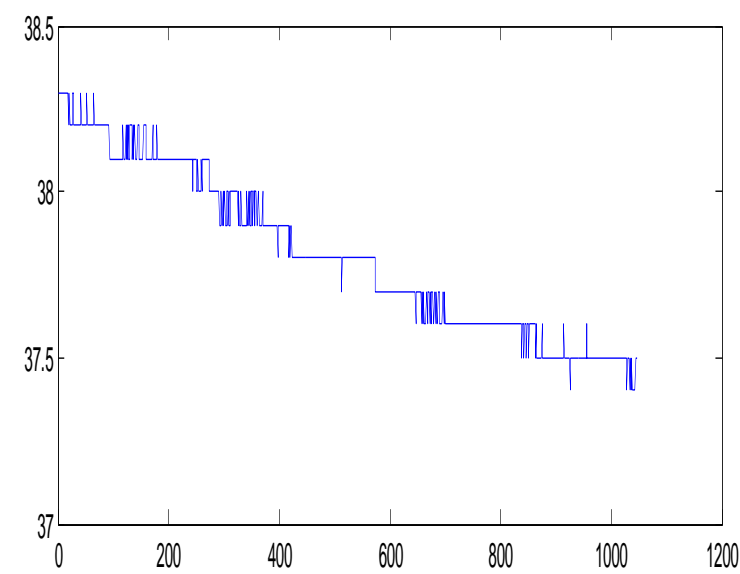

Figure 2: Measure of the temperature in the incubator without heating When the incubator is not heated, the model remains the same with a change of the parameters: $\mathrm{K}=-0.9$ and $\tau=680$.

\section{ARCHITECTURE OF THE BOARD}

We developed a board for acquisition and control which requires a tool for its digital processing. For this we used a microcontroller (PIC 16F877), given its good reputation in the industrial and automatism sector. We also need some output devices as the 7 -segment displayers, a power supply, keyboard, temperature sensors, a control block of thermal resistance and finally a communication network.

The general architecture of the acquisition and control board for incubator is presented by the diagram below:

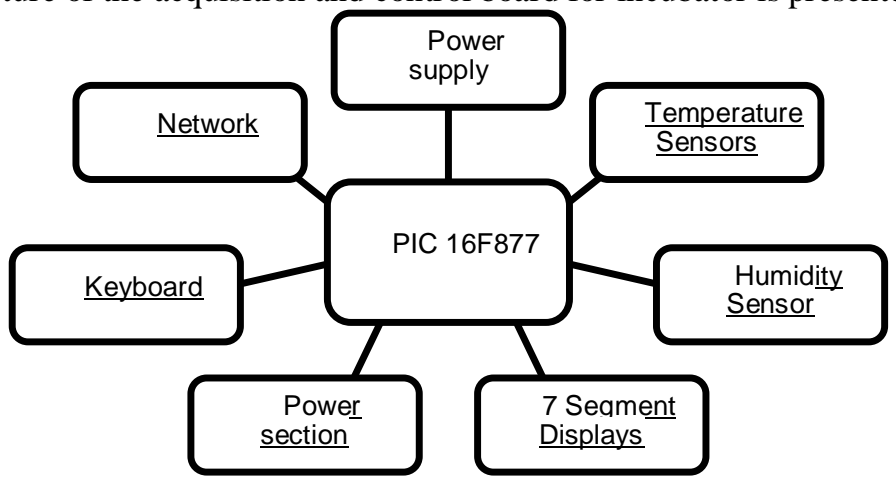

Figure 3 : Block diagram of the card 


\section{Dual Redundancy}

\section{REDUNDANCY TECHNIQUES}

The redundancy provides effective protection against random component failures. It is commonly used for systems including the critical functions due to the increased cost (equipment, installation, maintenance, weight and power). In the majority of redundant facilities we use the double or triple redundancy. The dual redundancy is used when we only need to detect the sensor failure, but the triple redundancy could be used for more purposes. The redundancy of high order is reserved for complex applications that require high performance and to which access is difficult and expensive as it is the case of a space vehicle. Each of these can be implemented in static or dynamic redundancy.

In our case we focus in measuring the temperature inside the incubator. We used two identical linear type of sensors LM35, the same chain of conditioning and the acquisition which is done in the same converter built into the PIC 16F877. This latter has eight multiplexed analogical inputs. Once the acquisition is done, we obtain the following shape:

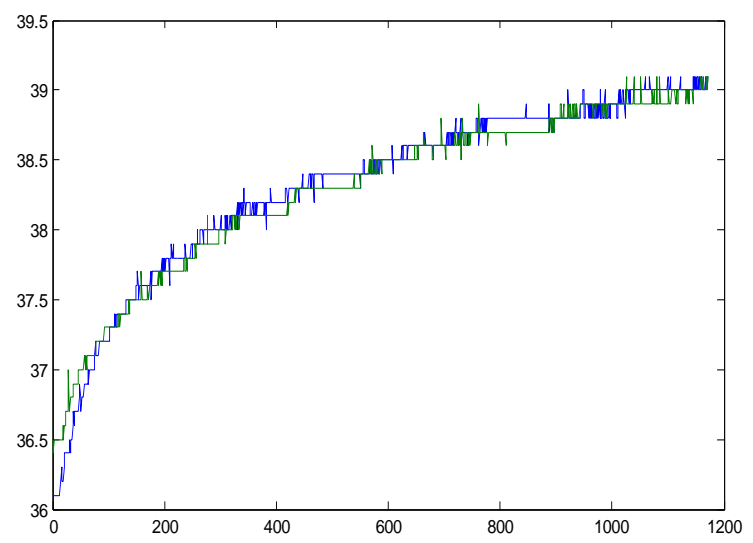

Figure 4 : Temperature measurements during heating

The voting system used in most dual redundant analogical system is the average. The results are shown in the figure below:

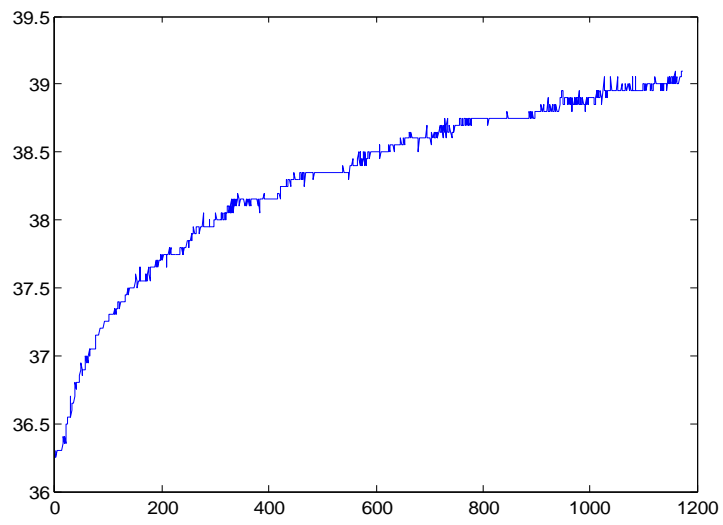

Figure 5 : The shape of the acquisition

Systems working with small errors are effective ones. However, if one sensor is disconnected, the system will not be able to resolve the problem adequately. The system response is illustrated by the following two graphs: 


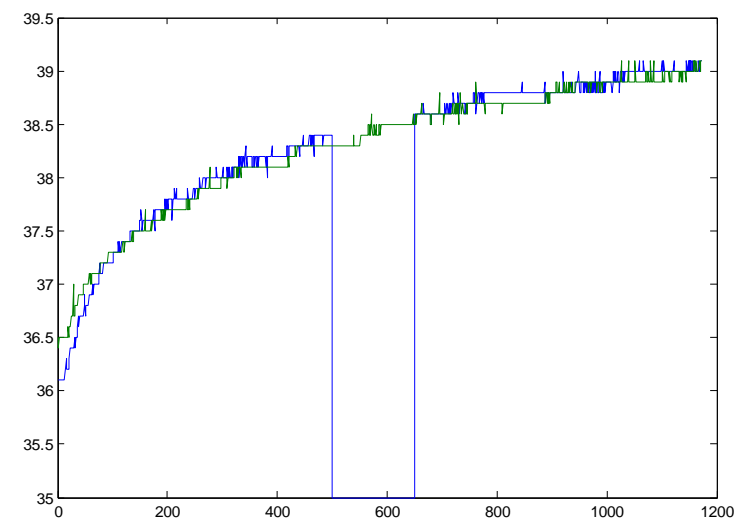

Figure 6 : acquisition (sensor 2 fault)

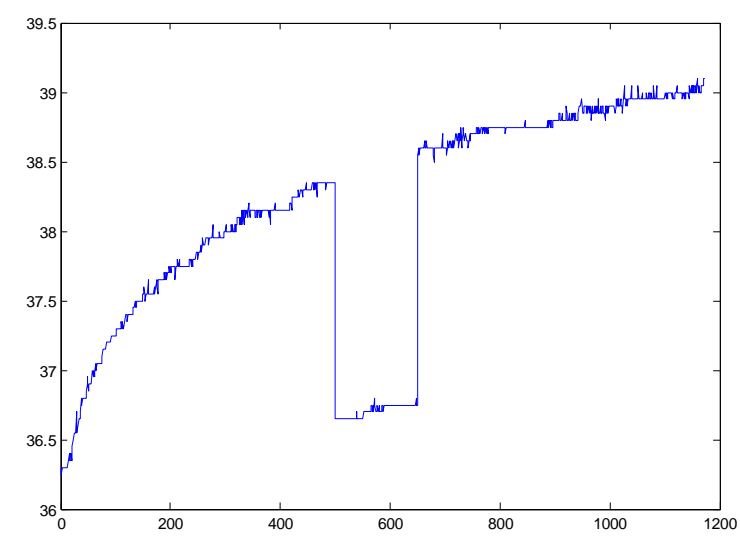

Figure 7 : Shape of for acquisition (sensor 2 fault)

The dual redundancy is architecture with two different usage and provides a "self-use" vote to reduce transitory events. Hence, this latter technique could be used as a noise filter.

\section{Time redundancy}

We know that the temperature in the incubator varies very slowly and is almost constant during one second. For this, the final decision at the output level of our system will be influenced by the output of both sensors at time $\mathrm{t}$ and time $\mathrm{t}-1$.

$$
S(t)=\frac{C_{1}(t)+C_{2}(t)+C_{1}(t-1)+C_{2}(t-1)}{4}
$$

C 1: Sensors at temperature 1

C 2: Sensors at temperature 2

In the time redundancy, voting time is used instead of spatial redundancy. The combination of dual redundancy and self-electors can provide a good performance.

Such redundancy eliminates a fault that occurs during a short time. We can notice below the influence of the time redundancy on the system output.

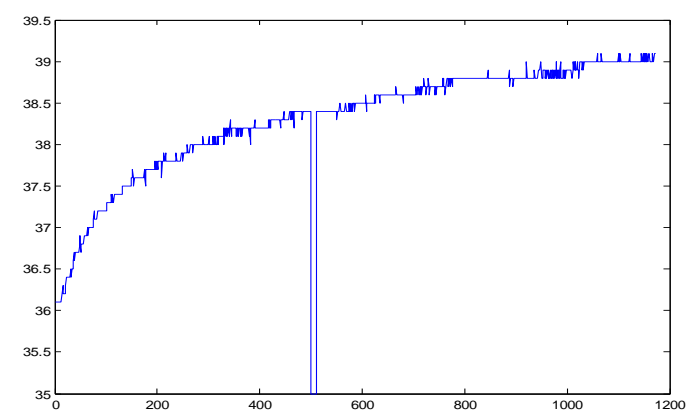

Figure 8 : acquisition (sensor fault) 


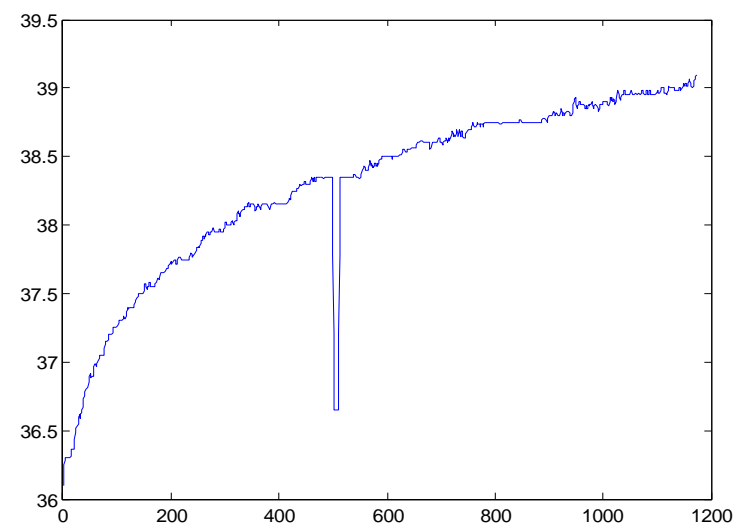

Figure 9 : shape for acquisition with time redundancy

\section{Triple redundancy}

Security and user-friendly aspect are the benefits of new systems during the last ten years. The challenge, however, remains in the reliability and fault tolerance in the systems. The most tolerant control system of faults requires redundancy in the hardware to make it more reliable. However, the additional components used for hardware redundancy increases its cost. The concept of analytical redundancy has been designed to replace some equipment especially in aerospace applications, reducing the overall cost and improving its reliability.

The theoretical methods of analysis of redundancy have been studied in the drive systems for aerospace applications up to today.

We first developed a model for the temperature in the incubator to enable then the development of methodologies for analyzing redundancy. Based on the model of an incubator system, an estimator is designed to assess the current temperature in the incubator. The observed value is then used as a sensor for analyzing the reading step and to replace one of two sensors of the redundant hardware.

The algorithms used for fault detection, isolation, and accommodation were also developed, based on the voting algorithm for the minimization of errors.

To develop the analytical redundancy, we must first understand the dynamics of the steering system. This dynamics can be approximated by the equations of the first orders described above.

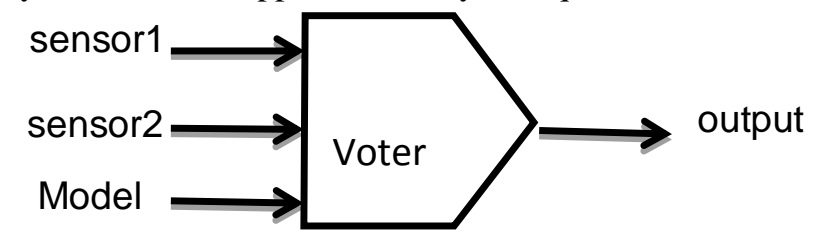

Figure 10 : voting system

he voting system calculates the absolute differences between each pair of entries. These differences are called residuals.

$\mathrm{R} 1=\mid$ sensor 1 - sensor $2 \mid$

$\mathrm{R} 2=\mid$ sensor 1-Model $\mid$

$\mathrm{R} 3=\mid$ sensor 2-Model $\mid$

The smallest residue is the best measure. For this we take as output the average of theses values. This technique provides a good decision even if one sensor fails as shown in the graph below: 


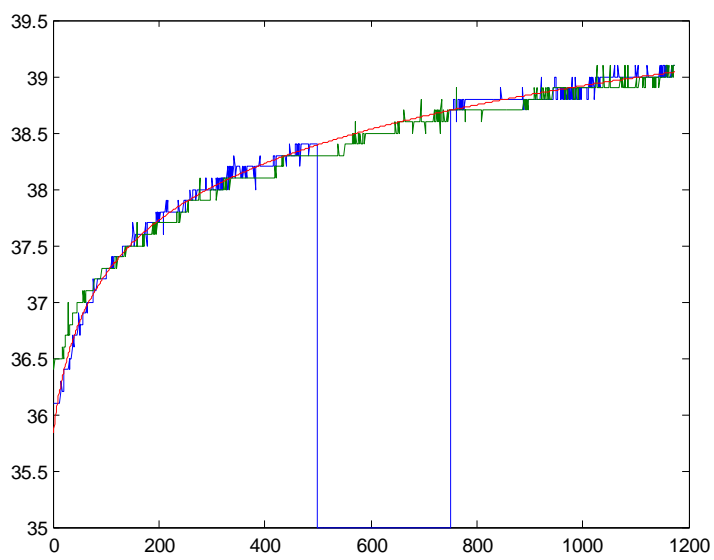

Figure 11 : acquisition (sensor2 fault) + model

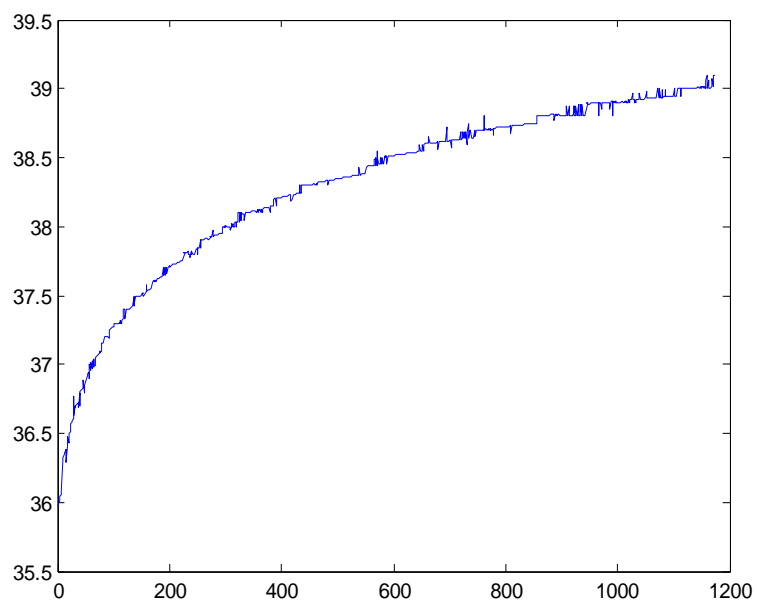

Figure $12:$ Shape of the acquisition with triple redundancy

\section{Triple redundancy and time redundancy}

The time redundancy and analytical one can provide alternative solutions which are less costly than hardware redundancy for applications where a delay or loss of accuracy can not be tolerated.

The system opted for the reliable measurement of temperature is the integration of hardware redundancy with a dual analytical redundancy and time redundancy. The solution is a dual quad architecture jobs and self-use voting to reduce transitory events and faults of this sensor and to ensure reliability. It uses the same algorithm as for the triple redundancy taking into account the last value generated by the voter.

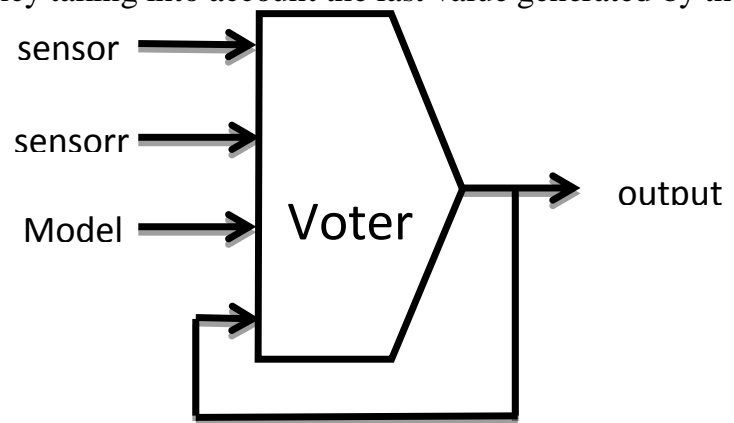

Figure 13 : triple redundancy voter with temporal redundancyThe output becomes: 


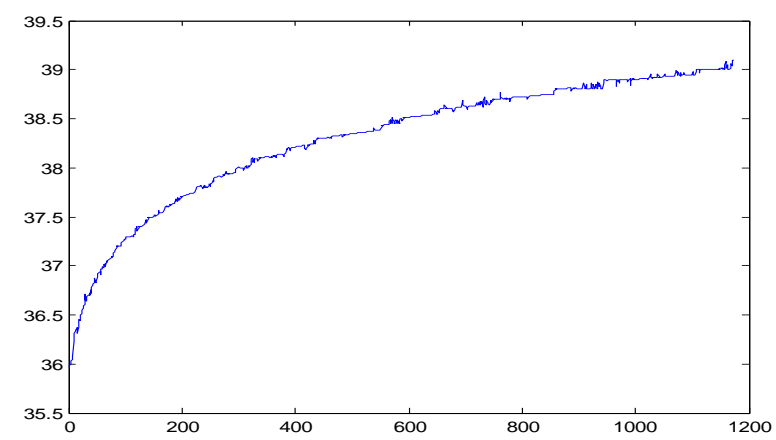

Figure 14 : Output of triple redundancy voter with temporal redundancy

\section{PROCESS MONITORING}

When calculating the final value, the voter also calculates the number of times the sensor 1 is used for voting the same for the sensor2. A fault is reported on the operation of the sensor which is rarely used in the calculation of the output. Our system is able to carry using the incubator in a proper way even if one sensor fails, while providing a filter for the sensor's output.

\section{CONCLUSION}

Dependability is realized by exploiting the redundancy of the sensors in the case of an application on the error is not tolerated. The treatment method of redundancy used for reliable measurement of temperature in an incubator is the hardware redundancy with dual analytical redundancy

\section{REFERENCES}

[1] Syed Askari, Badri Dwivedi, Adnan Saeed et Mehrdad Nourani (2010), Scalable Mean Voter for Fault-Tolerant Mixed-Signal Circuits, IEEEAC Paper 10 (page 1-10)

[2] Jian-xiao Zou, Hong-bing Xu (2009) IEEE Design and Reliability Analysis of Emergency Trip System with Triple Modular Redundancy (page 1006-1009)

[3] Albert Myers (2008), Achievable Limits on the Reliability of k-out-of-n: G Systems Subject to Imperfect Fault Coverage, IEEE Transactions On Reliability 57 (page 349-354)

[4] Huseyin Aysan, Sasikumar Punnekkat, and Radu Dobrin(2008) VTV - A Voting Strategy for Real-Time Systems, 2008 14th IEEE Pacific Rim International Symposium on Dependable Computing (page 56 -63)

[5] Albert Myers, and Antoine Rauzy(2008), Efficient Reliability Assessment of Redundant Systems Subject to Imperfect Fault Coverage Using

[6] Mehrdad Nourani, Ali Namazi and Syed Askari (2008), Fault Tolerant Circuits for Highly Reliable Systems, IEEEAC Paper \#1746, (page 1-10)

[7] John Teifel (2008), Self-Voting Dual-Modular-Redundancy Circuits for Single-Event-Transient Mitigation, IEEE Transactions On Nuclear Science 55 (page 3435-3439)

[8] Sohel Anwar and Lei Chen (2007), An Analytical Redundancy-Based Fault Detection and Isolation Algorithm for a Road-Wheel Control Subsystem in a Steer-By-Wire System, IEEE Transactions On Vehicular Technology 56 (page2859-2869)

[9] Dejan Desovski, Yan Liu and Bojan Cukic,Ninth(2005), Linear Randomized Voting Algorithm for Fault Tolerant Sensor Fusion and the Corresponding Reliability Model, IEEE International Symposium on High-Assurance Systems Engineering 10 (page1-10)

[10] Sherif Yacoub, Xiaofan Lin, Steve Simske, John Burns(2003), Automating the Analysis of Voting Systems, International Symposium on Software Reliability Engineering (ISSRE'03) 1-12

[11] Joakim Aidemark, Peter Folkesson, and Johan Karlsson (2003), On the Probability of Detecting Data Errors Generated by Permanent Faults Using Time Redundancy, Proceedings of the 9th IEEE International On-Line Testing Symposium (IOLTS'03)

[12] Hyunki Kim, Hyung-Joon Jeon, Keyseo Lee Kwangwoon, Hyuntae Lee Mokwon (2002), The Design and Evaluation of All Voting Triple Modular Redundancy System PROCEEDINGS, Annual RELIABILITY and MAINTAINABILITY Symposium (page 439 444)

[13] Joakim Aidemark, Jonny Vinter, Peter Folkesson, and Johan Karlsson(2002), Experimental Evaluation of Time-redundant Execution for a Brake-by-wire Application Proceedings of the International Conference on Dependable Systems and Networks (DSN'02)

[14] Mihaela Radu, Dan Pitica, PhD et Cristian Posteuca(2000), Reliability and Failure Analysis of Voting Circuits in Hardware Redundant Design, Int'l Symp on Electronic Materials \& Packaging 3 (page 421-423)

[15] Chung-Ho Chen(1999), Fault-Containment in Cache Memories for TMR Redundant Processor Systems, IEEE Computer Society, and Arun K. Somani, IEEE TRANSACTIONS ON COMPUTERS, VOL. 48, NO. 4, (page 386-397)

[16] Kalhee Kim, Mladen A. Vouk and David F. McAllister (1998), Fault-Tolerant Software Voters Based on Fuzzy Equivalence Relations ,IEEE (page 5-19)

[17] R. J. Patton (1991), Fault detection and diagnosis in aerospace systems using analytical redundancy COMPUTING \& CONTROL ENGINEERING JOURNAL (page 127-136)

[18] WIL J. VAN GILS (1986)A Triple Modular Redundancy Technique Providing Multiple-Bit Error Protection Without Using Extra Redundancy, IEEE TRANSACTIONS ON COMPUTERS, VOL 35, NO. 7, (page 623-631)

[19] STEPHEN Y. H. SU AND EDGAR DuCASSE (1980), A Hardware Redundancy Reconfiguration Scheme for Tolerating Multiple Module Failures. IEEE TRANSACTIONS ON COMPUTERS, VOL. c-29, NO. 3, MARCH (page 254-258)

[20] Norman G. Dennis (1974), Reliability Analyses of Combined Voting and Standby Redundancies, IEEE Transactions On Reliability 23 (page 66-75) 